\title{
A Dynamic Dynamometer for Testing of Mining DC Motors
}

\author{
R.E. Betz ${ }^{*}$, Galina Mirzaeva ${ }^{\dagger}$, T.J. Summers ${ }^{\ddagger}$ \\ School of Electrical Engineering and Computer Science \\ University of Newcastle, Australia, 2308 \\ email:*Robert.Betz@newcastle.edu.au; \\ ${ }^{\dagger}$ Galina.Mirzaeva@newcastle.edu.au; \\ †Terry.Summers@newcastle.edu.au
}

\begin{abstract}
This paper describes the development of a $2.2 \mathrm{MW}$ DC electric machine based dynamometer for the cyclic testing of motors used in mining applications. The dynamometer, through the application of sophisticated control, is able to simulate the real dynamic digging conditions of an electric rope shovel. The paper will discuss the facility and the control algorithms to implement the dynamic dynamometer. Simulation results will be presented.
\end{abstract}

\section{NOMENCLATURE}

$B_{d} \quad$ Drive machine self friction coefficient.

$B_{l} \quad$ Load machine self friction coefficient.

$B_{t} \quad$ Total dynamometer friction coefficient $\left(B_{d}+B_{l}\right)$.

$B_{e m} \quad$ Friction coefficient to be emulated.

$G(s)$ Transfer function of the coupled dynamometer machines.

$G_{\text {comp }}$ Load machine loop compensation transfer function.

$G_{c} \quad$ Speed controller transfer function.

$G_{h}(s)$ Continuous time transfer function for the sample hold.

$G_{t} \quad$ The speed controller transfer function for the load machine.

$G_{\mathrm{em}} \quad$ Emulated sysem transfer function.

$J_{b} \quad$ Base inertia.

$J_{d} \quad$ Drive machine self inertia.

$J_{l} \quad$ Load machine self inertia.

$J_{t} \quad$ Total dynamometer inertia $\left(J_{d}+J_{l}\right)$.

$J_{e m} \quad$ Inertia to be emulated.

$T_{b} \quad$ Base torque.

$T_{d} \quad$ Reference drive machine torque.

$T_{l} \quad$ Reference load machine torque.

$T_{s} \quad$ Controller sampling period.

$T_{t} \quad$ Total reference torque for the two machines - i.e. $T_{e}+$ $T_{l}$.

$T_{d_{\text {rated }}}$ Drive machine rated torque.

$T_{l_{\text {rated }}}$ Load machine rated torque.

$T_{n_{d}} \quad$ Normalised drive machine torque.

$T_{n_{l}} \quad$ Normalised load machine torque.

HECS Hall Effect Current Sensor.

HEFS Hall Effect Flux Sensor.

RTD Resistive Thermal Device.

\section{INTRODUCTION}

Large electrical machines are used extensively in the mining industry, in applications from mine shaft hoists to draglines. These DC machines are often subject to very harsh operational conditions, both from a physical viewpoint with respect to things such as ambient temperature, dust etc., and also from operational stress as they are often being used with highly dynamic loads, and consequently subject to constant large magnitude high rate of change currents. This puts a lot of stress on the commutator and brushes of the machine.

Industry in general has tended to move towards using $\mathrm{AC}$ induction machines because of their robustness and low maintenance. However, DC motors are still used extensively in open cut mining equipment such as rope shovels and draglines (although the very latest equipment is now using AC motors). The persistence of DC motors in the mining industry, despite the trend towards AC machines in general, is largely to do with the fact that the industry has had a lot of experience with DC motors, and understand their limitations, performance, reliability, and how to maintain them. Furthermore, the control for these machines is very simple, and the power electronics used in the drives is robust relative to that used in AC drives. Therefore, even though the DC motor share of the mining market is decreasing, they will be in service for many years to come.

DC motor performance requirements in mining applications will continue to grow due to increasingly difficult digging conditions and production pressures. Meeting these production imperatives not only requires the machines to perform at their design limits (and sometimes beyond), but also requires high reliability and availability. These requirements are in juxtaposition to each other. In order to simultaneously achieve high performance and reliability it is important to thoroughly understand the performance limitations of these machines.

A project has been established between the University of Newcastle, Australia, and industry partners that has included the development of a full size DC motor testing facility. This facility is designed to allow the full load testing of the DC motors used in electric rope shovels. It allows for both static and dynamic testing, and has the following features: 
- Two industrial DC motors of the same size connected back-to-back, one being the test motor, the other working as a programmable load (these roles can be swapped);

- Two sets (one for each motor) of the following: a thyristor converter, digital drive, reactive power compensator and other devices that are normally used with rope shovel DC motors;

- A large number of sensors installed internally and externally on the motors to collect information about the machine's electrical, mechanical and magnetic performance;

- PC-based data acquisition system to record, process and visualise experimental data;

- Custom written signal processing software to analyse the collected data and extract relevant information about the performance of the machines.

Remark 1: The use of recently available instrumentation in this facility allows the measurement of various parameters in the machine, such as the flux density distribution across the main pole and the interpoles of the machines. Previously sensors were not available that could fit in the air gap of the electrical machines, and consequently the measurements taken in this paper could not be undertaken.

The remainder of this paper is organised as follows. Section II outlines the physical structure of the test facility, including the machines and instrumentation. Section III presents details of the dynamometer control algorithm. Section IV presents the results from the simulation studies of the control algorithm. Finally Section V presents conclusions and the contributions made by this paper.

\section{THE TEST FACILITY}

The dynamometer test facility has been constructed on a mine site in the Hunter Valley, NSW Australia. Fig. 1 is block diagram of the structure of the facility.

One can see that there are two DC machines with their shafts connected together via a torque transducer and coupling. One machine is considered to be the drive or test machine and the other a load machine. The two machines used, whilst of similar rating, are actually different motors produced by different manufacturers. Because the instrumentation and control equipment is identical for each motor, the role of test machine and load machine is interchangeable. This allows a comparison of the performance of the two machines, which have slightly different designs.

Both machines have had a lot of instrumentation installed in them prior to being assembled at the facility - e.g. flux sensors on the pole faces, temperature sensors, various current sensors, mechanical vibration sensors, and so on. Two PCs with data acquisition boards are used to collect the sensor outputs, and the data is saved in files for future off-line signal processing. The two machines are controlled by ABB DCS600 ${ }^{\circledR}$ thyristor based DC drives.

The signals measured on the machines are shown in Fig. 2. As can be seen from this figure the measurements are very comprehensive. As mentioned above, the PCs have high speed A/D cards installed, and custom software had to be written to

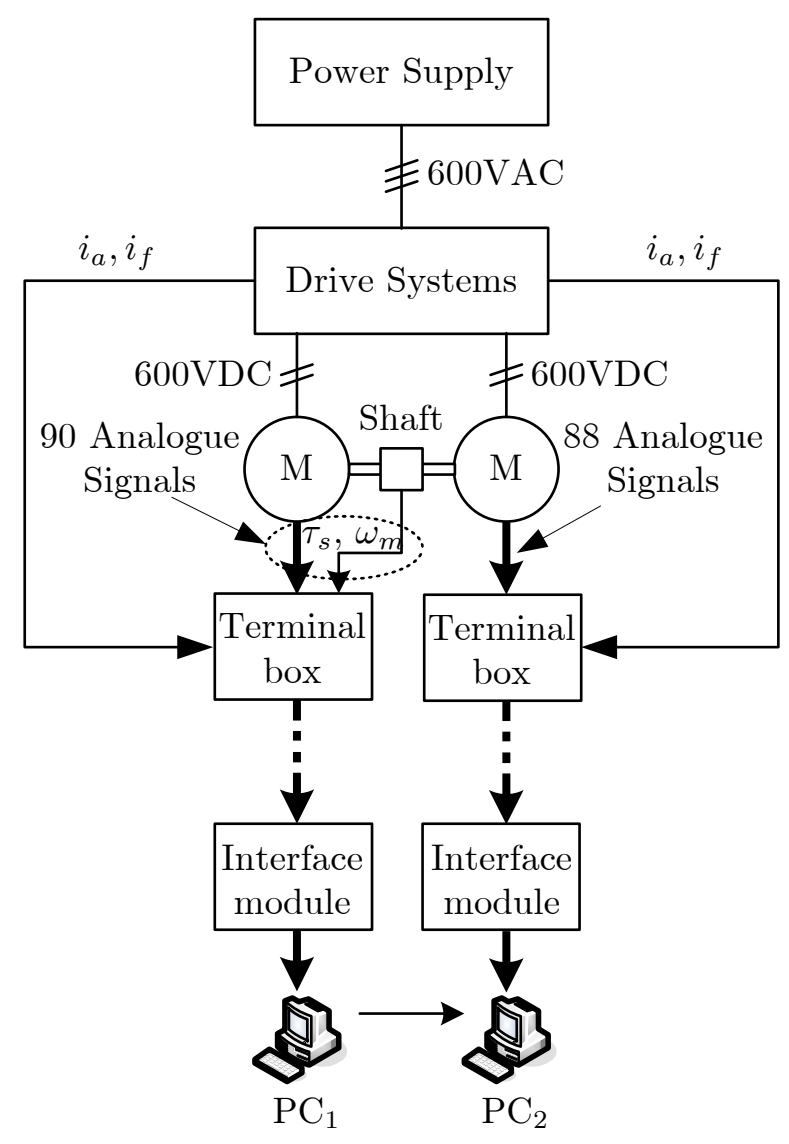

Fig. 1. Block diagram of the dynamometer facility.

handle the volume and speed of the data from the large number of channels present.

Hall Effect Flux Sensors (HEFSs) are mounted on the pole faces of the machines. The sensors are very small so that they can be glued to the pole faces of the machine without hitting the rotor. Arrays of sensors are fitted to the main field pole (which also includes a compensating winding), the interpoles, and on a non-metallic bridge between the interpoles and the field windings. The sensors used are capable of reading flux densities up to approximately 1.8 Tesla. They all had to be calibrated prior to mounting in the machines. The Resistive Thermal Devices (RTDs) are similarly mounted, and are used to measure the internal operating temperature of the machine. Fig. 3 shows the mounting points of the HEFSs and RTDs in one of the machines. Note the non-metallic epoxy bridge in the main field/interpole gap so that HEFSs can be mounted there. The main pole sensors are mounted in between the compensating winding slots. The wiring used has high temperature insulation and is shielded twisted pair so that noise pick-up is minimised.

The armature and field currents and voltages are also measured. The armature current measurements are carried out using three instruments - a Hall Effect Current Sensor (HECS) for the total current, a Rogowski coil, and pigtail HECSs on the leads to the individual brushes in some of the brush arms. Two 


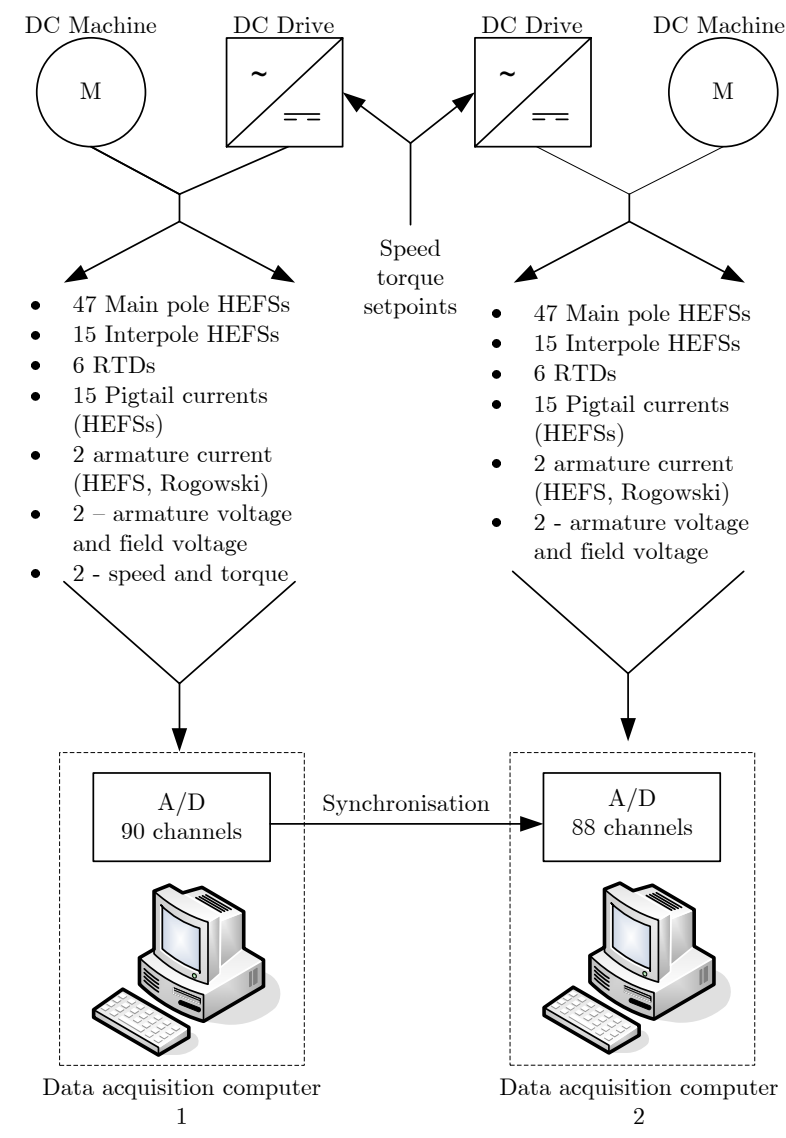

Fig. 2. Block diagram showing the signals measured on the dynamometer.

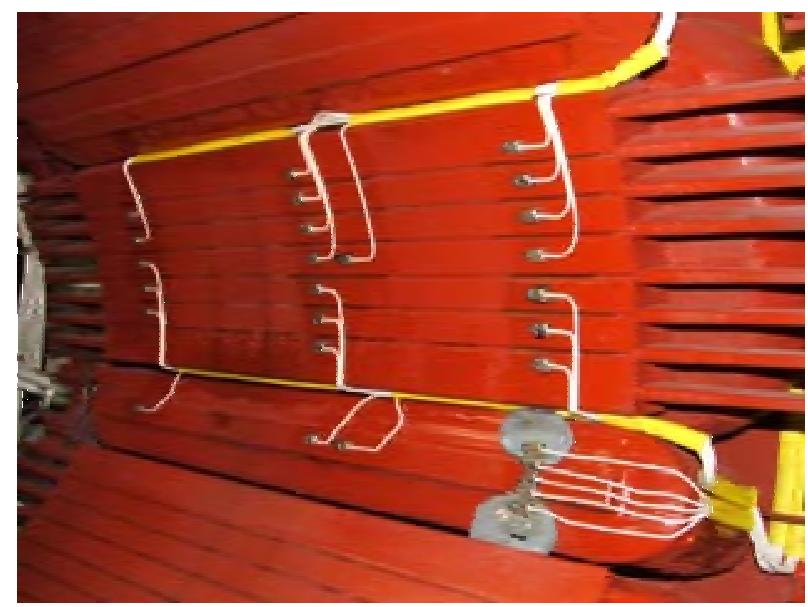

Fig. 3. Mounting points for the HEFSs and the RTDs in one of the DC machines.

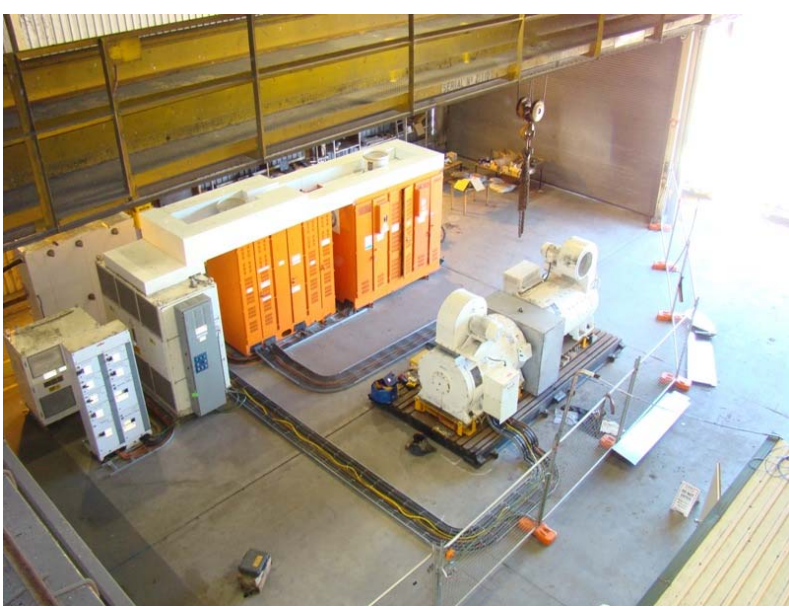

Fig. 4. Picture of the dynamometer system.

armature current sensors are used to cover different frequency ranges in the currents - the HECS only cover frequencies from zero to approximately $100 \mathrm{kHz}$, and the Rogowski coil covers from $100 \mathrm{kHz}$ to approximately $100 \mathrm{MHz}$. The high frequency current spectrum is of interest because these frequencies are present when there is sparking between the commutator and the brushes.

Fig. 4 is a photograph of the experimental facility . One can see the two back-to-back DC motors, the drive cabinets (orange), and the circuit breakers (the cabinets on the left).

\section{DYNAMIC DYNAMOMETER CONTROL}

It was intended to use the dynamometer for both static and dynamic testing of the DC motors. Static testing, and some basic dynamic testing have been carried out by using the speed loop of the test machine to keep its speed constant. The load machine has step changes of the reference speed input into its speed loop, but the torque limits are set lower than the test machine so that the test machine is able to keep its speed constant. Using this approach one can generate step changes in the load torque on the test machine. Clearly this type of test is a constant speed test, and does not emulate conditions experienced during mine digging cycles. Results of this testing are described in more detail in [5], [6].

In order to emulate digging the load machine needs to be controlled so that it emulates the dynamics of the digging operation - i.e. the appropriate inertia, friction coefficients, as well as impulse and static load torques. There are several ways of constructing the control to allow such emulation. If one wishes the control of the load machine to be totally independent of the test machine (which means that the load machine is essentially a self contained load emulator), then high bandwidth measurements of the shaft torque are required. Furthermore, the control usually involves the use of derivatives, and therefore can be compromised if there is noise on the measurements [3], [7], [9]. An alternative approach, which does not require the use of derivatives and does note require a torque transducer, is based on controlling the torques of both 


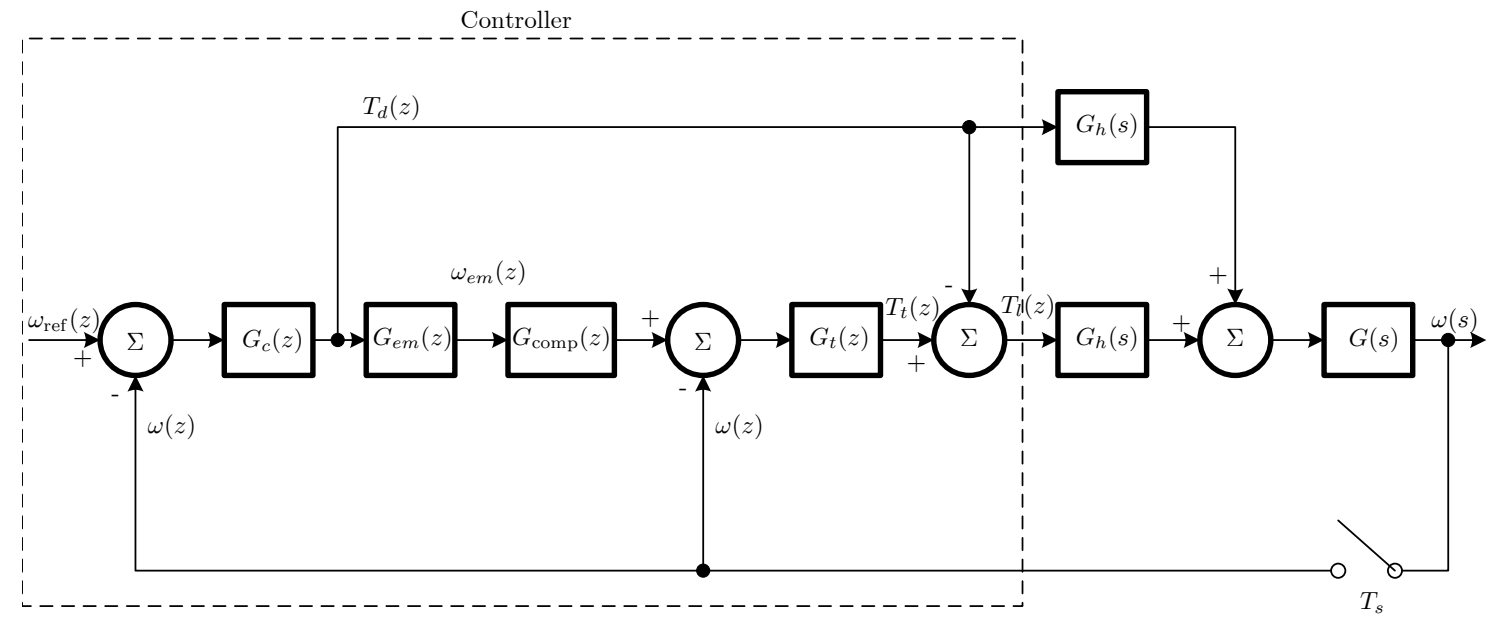

Fig. 5. Block diagram of the control scheme [4].

of the machines so that the correct emulation is obtained [1], [2], [4].

In the following discussion $G_{c}$ is the test machine speed controller transfer function, $G_{\mathrm{em}}$ is the transfer function for the dynamics of the system to be emulated, $G_{\text {comp }}$ is a loop compensation transfer function, $G_{t}$ is the speed controller transfer function of the load machine, $G_{h}(s)=\frac{1-e^{-s T_{s}}}{s}$ is the transfer function of a sample hold, and finally $G(s)$ is the transfer function for the coupled dynamometer machines. An ' $s$ ' in the transfer function means a continuous time Laplace transform transfer function, and a ' $z$ ' means a discrete time $Z$ transform transfer function.

Fig. 5 is a block diagram of the control strategy employed for this system [1]. Note that the machine current dynamics are assumed to be much faster than the load dynamics and therefore have been ignored in this model - i.e. the electrical machines are considered to be a pure algebraic torque source.

The basic idea behind this control strategy is that the drive machine speed controller produces a desired drive machine torque $T_{d}$ based on the error between the drive machine speed and the desired speed. This is the torque that will be specified as a reference to the drive machine torque control loop. The same torque is also applied to the load dynamics emulation model $\omega_{\mathrm{em}}(z)=G_{\mathrm{em}}(z) T_{d}(z)$. This produces the speed that the emulated system would rotate at under this torque - i.e. $\omega_{\mathrm{em}}$. Ignoring $G_{\mathrm{comp}}(z)$ for the moment, this speed becomes the reference for the dynamometer speed control loop. The $G_{t}(z)$ controller in this loop produces the torque $T_{l}$ that would be required to make the actual dual machine dynamics rotate at $\omega_{\mathrm{em}}$. This torque is the total torque required for the two machines - i.e. the total torque is the addition of the drive and load machine torques. Therefore the contribution that the load machine must make towards this total torque is $T_{l}=T_{t}-T_{d}$, since the drive machine is going to produce torque $T_{d}$. Clearly if the drive machine is producing a torque of $T_{d}$, which is the torque required to make the drive machine move towards the desired reference speed, but the actual shaft speed of the drive machine is responding as though this torque is being applied to the $G_{\mathrm{em}}$ transfer function, then the dynamics seen by the drive machine must be $G_{\mathrm{em}}$.

Remark 2: One can see that this control strategy involves well known control techniques, and does not require the use of derivatives or torque measurements. However, it does mean that the load machine and the drive machine become a tightly coupled control system, and the load machine is not a stand alone arbitrary load emulation system.

The compensation function $G_{\mathrm{comp}}(z)=\frac{1+G_{t}(z) G(z)}{G_{t}(z) G(z)}$ is the inverse transfer function of the inner load machine speed loop - i.e the effective dynamics of the blocks to the right of $G_{\text {comp }}(z)$ in Fig. 5. If this inverse function is ideal then the $\frac{\omega_{\mathrm{em}}(z)}{\omega(z)}=1$ and the overall loop would look like Fig. 6, which means that the dynamics of the system become $\frac{\omega_{\mathrm{em}}(z)}{T_{d}(z)}=G_{\mathrm{em}}(z)$ as required.

Now let us consider the control in more detail. The speed control transfer functions $G_{c}(s)$ and $G_{t}(s)$ are standard PI controllers - i.e.:

$$
G_{\mathrm{pi}}(s)=\frac{K_{p}\left(s+\frac{1}{T_{i}}\right)}{s}
$$

Using the Tustin or bi-linear approximation [8] this can be written in discrete form as:

$$
G_{\mathrm{pi}}(z)=\frac{s_{0} z+s_{1}}{z-1}=\frac{s_{0}+s_{1} z^{-1}}{1-z^{-1}}
$$

where:

$$
\begin{aligned}
& s_{0}=K_{p}\left(1+\frac{T_{s}}{2 T_{i}}\right) \\
& s_{1}=K_{p}\left(-1+\frac{T_{s}}{2 T_{i}}\right) \\
& T_{s} \triangleq \text { the sampling period }
\end{aligned}
$$

The load dynamics to be emulated are the traditional rotating load dynamics, which can be expressed as a continuous 
time transfer function as:

$$
G_{\mathrm{em}}(s)=\frac{1}{J_{\mathrm{em}} s+B_{\mathrm{em}}}
$$

and can be translated into discrete form (again using the bilinear approximation) as:

$$
G_{\mathrm{em}}(z)=\frac{T_{s}(z+1)}{\left(2 J_{\mathrm{em}}+T_{s} B_{\mathrm{em}}\right) z+\left(T_{s} B_{\mathrm{em}}-2 J_{\mathrm{em}}\right)}
$$

Remark 3: Equation (6) is representative of the general dynamics of loads in mining applications since the digging arm is reflected through the gearbox as an inertia and a speed dependent friction coefficient. The parameters in the equation would be changing dependent on the dipper load and the geometry of the shovel arm extension. An added part of the emulation would be impact forces as reflected back to the DC machine shaft through the gearbox. These would appear as step disturbance torques. This effect can be emulated by subtracting the transient shaft torques from $T_{d}(z)$ prior to the torque input into the $G_{\mathrm{em}}(z)$ transfer function. This will result in a transiently lower or higher emulation speed reference depending on the sign of the transient torque (it would usually be a negative torque resulting in a lower emulation speed reference.)

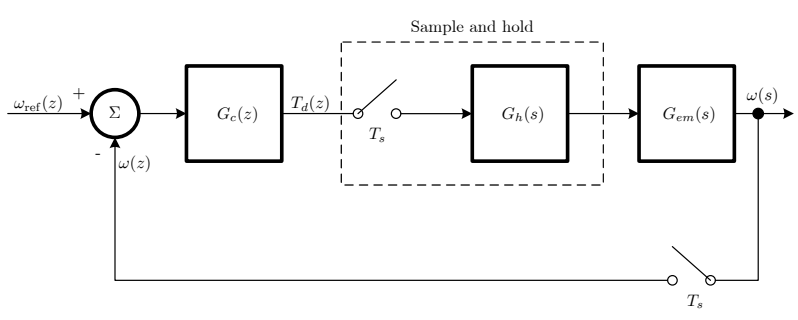

Fig. 6. Block diagram of control with ideal compensation [4].

The next transfer function in the system is for the dynamometer natural dynamics. As mentioned previously, the electrical dynamics are at least an order of magnitude faster than the self mechanical dynamics of the dynamometer and the emulated load dynamics. Therefore, as a first approximation, these are ignored. Hence the dynamics of the system become:

$$
G(s)=\frac{1}{\left(J_{d}+J_{l}\right) s+\left(B_{d}+B_{l}\right)}=\frac{1}{J_{T} s+B_{T}}
$$

where $J_{d}, J_{l}, B_{d}$ and $B_{l}$ are the drive and load machine rotational inertia and friction coefficients respectively. Similarly to the emulation function, the discrete form of (8) is:

$$
G(z)=\frac{T_{s}(z+1)}{\left(2 J_{t}+T_{s} B_{t}\right) z+\left(T_{s} B_{t}-2 J_{t}\right)}
$$

where $J_{t}=J_{d}+J_{l}$ and $B_{t}=B_{d}+B_{l}$.

We are now in a position to define $G_{\text {comp }}(z)$ :

$$
\begin{aligned}
G_{\mathrm{comp}}(z) & =\frac{1+G_{t}(z) G(z)}{G_{t}(z) G(z)} \\
& =\frac{A z^{2}+B z+C}{T_{s} s_{0 t} z^{2}+T_{s}\left(s_{0 t}+s_{1 t}\right) z+s_{1 t} T_{s}}
\end{aligned}
$$

where:

$$
\begin{aligned}
& A=2 J_{t}+T_{s} B_{t}+T_{s} s_{0 t} \\
& B=T_{s}\left(s_{0 t}+s_{1 t}\right)-4 J_{t} \\
& C=2 J_{t}-T_{s} B_{t}+T_{s} s_{1 t}
\end{aligned}
$$

\section{A. Emulation Limitations}

All dynamometers that emulate loads have limitations on the systems that can be emulated determined by the torque capabilities of the load machine. The dynamometer, which is the subject of this paper, has two virtually identical machines for the drive and load (i.e. very similar power, torque, $J$ and $B)$. This fact means that there are even more severe limitations on what can be emulated as compared to the case where the load machine has larger torque and power output relative to the drive machine.

We shall simplify the situation so that it is easier to analyse the limitations. Consider the emulated load to have the following form:

$$
G_{\mathrm{em}}(s)=\frac{1}{J_{e m}}
$$

With this emulation transfer function, and if the torque rating of the machines is the same - i.e. $T_{d_{\text {rated }}}=T_{l_{\text {rated }}}$, we can make the following observations:

- The minimum emulated inertia is $J_{\mathrm{em}}=J_{d}$. Under this condition $T_{l_{\text {rated }}}=T_{d_{\text {rated }}}$ so that $\dot{\omega}=\frac{1}{J_{d}}$. In other words the torque of the load machine is all being used to accelerate its own self inertia, and therefore the torque of the drive machine is being used to accelerate its own self inertia.

- The maximum emulated inertia is infinity. This occurs when the torques produce by the drive and load machines are equal and opposite in sense. Therefore there is no resultant torque on the system and $\dot{\omega}=0$.

Under the condition of identical machines, let us define some bases as follows: $J_{d}=J_{l}=J_{b}$, and $T_{d_{\text {rated }}}=T_{l_{\text {rated }}}=T_{b}$. These allow the emulated inertia and torques to be expressed in pu form as:

$$
\begin{aligned}
J_{n} & =\frac{J_{\mathrm{em}}}{J_{b}} \\
T_{n_{d, l}} & =\frac{T_{d, l}}{T_{b}}
\end{aligned}
$$

Using these expressions and the dynamic equations for each machine and the dynamometer as a whole it can be shown that for a particular desired emulation inertia $J_{n}$ that the load machine torque is:

$$
T_{n_{l}}=\left[\frac{2-J_{n}}{J_{n}}\right] T_{n_{d}}
$$

This can be rearranged with $T_{n_{l}}=1$ to give the maximum drive machine torque that can be applied for a given emulation inertia so that the load machine can produce enough torque to faithfully produce the emulation:

$$
T_{n_{d}}=\left[\frac{J_{n}}{2-J_{n}}\right]
$$


Remark 4: Note that if $J_{n}=2$ that $T_{n_{d}}=\infty$. Under this condition the load machine torque is zero. The drive machine is driving its own self inertia and that of the load machine. Therefore the load machine torque limits do not matter, and the system performance is only constrained by the drive machine.

Example 1: Let $J_{n}=0.8$, which means that we are trying to emulate an inertia that is lower than the drive machine self inertia. Therefore from (16) we have:

$$
T_{n_{d}}=\frac{0.8}{2-0.8}=0.6667
$$

Therefore, under these conditions, the drive machine torque has to be limited to $0.66667 \times T_{b}$ for the load machine torque to stay within the $T_{b}$ limit and the load to be accurately emulated.

Example 2: If one wants to have transient load torques applied to the drive machine, and assuming that the load machine torque output is to be kept below $T_{l_{\text {rated }}}$ then the transient load changes have to be subtracted from the maximum load torque available. If this is done then the system dynamics will be faithfully reproduced even under transient torque inputs.

Assume $0.3 \mathrm{pu}$ transient load torques. Therefore the maximum steady state load machine torque available is $\tau_{n_{l}}=$ $0.7 \mathrm{pu}$. If we assume that we wish to emulate an inertia of $J_{n}=1.2 \mathrm{pu}$, then we have a maximum $\tau_{n_{d}}$ of:

$$
\tau_{n_{d}}=\left[\frac{1.2}{2-1.2}\right] 0.7=1.05
$$

Notice that this expression says that the drive machine torque can be larger than the rated torque if the load machine is producing rated torque. The reason for this is that the torque requirements from the load machine under this regime of operation are not great. So in this case equation (15) is the equation of interest. Therefore if the $T_{n_{d}}$ is allowed to be ' 1 ' and $J_{n}=1.2$ then:

$$
T_{n_{l}}=0.666667 \mathrm{pu}
$$

\section{Simulation Results}

To initially test the control strategy several simulations were constructed. The first was developed in Saber ${ }^{\circledR}$ as a proof of concept. This simulation was for the ideal situation portrayed in Fig. 5 which assumed that the machine is an algebraic torque source, and therefore only the load was simulated as a transfer function. The parameters used in this simulation study were arbitrary in nature, since this was simply a proof of concept. This simulation ran successfully, with the dynamics of the emulated system successfully followed. A second and more interesting variant of this Saber ${ }^{\circledR}$ simulation was constructed with a full model of the two DC machines and their control loops. Incidentally, the control in both these simulations was implemented in discrete time, and the load and machine dynamics were simulated in continuous time. Fig. 7 is the diagram of the simulation drawn in the Saber ${ }^{\circledR}$ simulator.

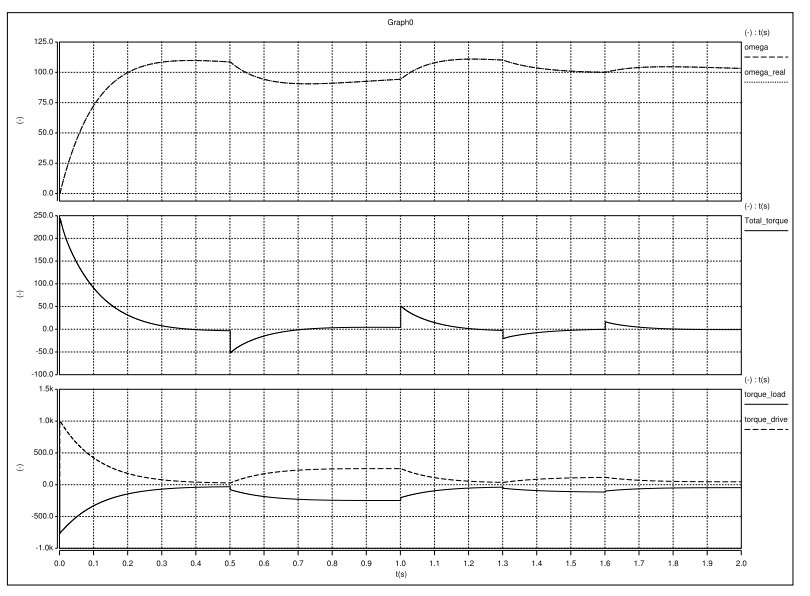

Fig. 8. Simulation plot from the Saber simulation including the machine dynamics and transient torques.

One can again see that it is very similar in structure to the control diagram of Fig. 5 except for the machine models and their associated current control loops.

A number of simulations were carried out using this simulation model to determine the performance of the controller. One set of results appear in Fig. 8. The load and machine parameters are not relevant with respect to the current discussion. In this simulation the overall drive machine controller was attempting to keep the speed constant at $100 \mathrm{rad} / \mathrm{sec}$ despite transient torques of $50 \mathrm{Nm}$ (at $0.5 \mathrm{sec}), 7 \mathrm{Nm}(1.0 \mathrm{~s})$, $80 \mathrm{Nm}(1.3 \mathrm{~s})$ and $12 \mathrm{Nm}(1.6 \mathrm{~s})$. The most important plot in the figure is the top one that shows the response of the exact emulation model (plot called omega_real), and the response of the dynamometer emulation of this model (plot called omega). Note that the two are virtually identical, indicating the precision of the emulation even under transient torque conditions and including the DC machine dynamics. The other two plots are the total dynamometer torque, and the two individual machine torques. It should be noted that the speed deviations away from the desired speed of $100 \mathrm{rad} / \mathrm{sec}$ are due to the poor tuning of the drive machine PI speed controller - it has very poor disturbance rejection.

The Saber ${ }^{\circledR}$ simulations, whilst useful for a proof-ofconcept, had certain limitations. For example, it was difficult to simulate the field weakening of the DC machines with the models in the simulator. Therefore the simulation was subsequently rewritten in the object oriented language called Python, which allowed the code to be much closer to that that would be implemented in the final experimental system. It is this code that has been used to produce the remaining plots.

Remark 5: The Python simulation is comprehensive in that it simulates not only the control and load dynamics as depicted in Fig. 5, but it also simulates the current loops within the thyristor based drives and the field weakening mode if the machines go above rated speed. It also allows multirate control, with the current and speed control loop rates to be set to those that will be used in the real experimental system. The parameters chosen for these results are those of the motors 


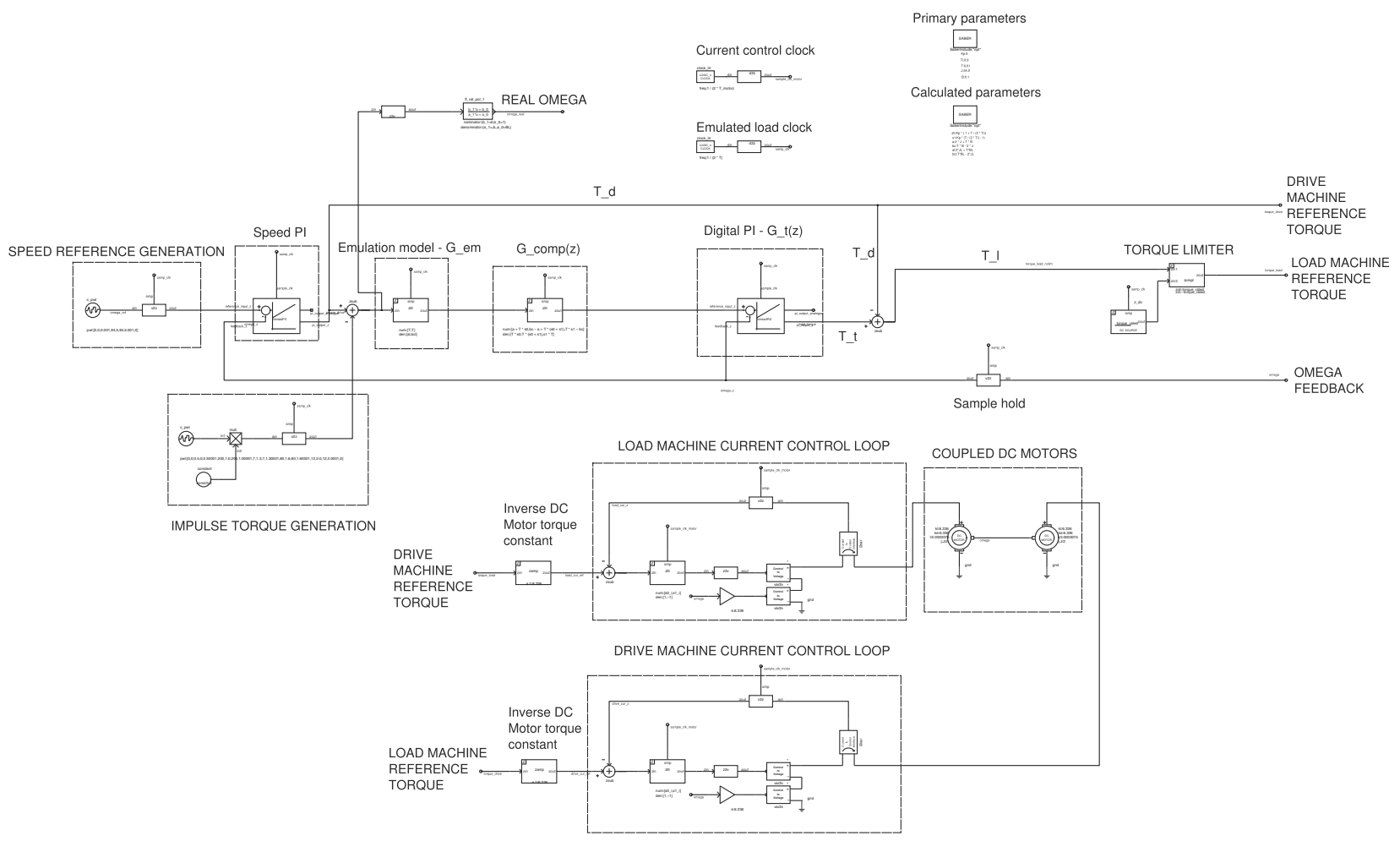

Fig. 7. Simulation of the dynamometer emulator from the Saber ${ }^{\circledR}$ simulator.

\begin{tabular}{|c|c|}
\hline Parameter Name & Value \\
\hline \hline$I_{a_{\max }}$ & $1925 \mathrm{Amp}$ \\
\hline$V_{a_{\max }}$ & $600 \mathrm{VDC}$ \\
\hline$\omega_{\text {rated }}$ & $94.25 \mathrm{rad} / \mathrm{sec}$ \\
\hline$P_{\text {rated }}$ & $1.155 \mathrm{MW}$ \\
\hline$T_{\text {rated }}$ & $12254.64 \mathrm{Nm}$ \\
\hline$J_{d, l}$ & $27 \mathrm{kgm}^{2}$ \\
\hline$L_{a}$ & $0.0003075 \mathrm{H}$ \\
\hline$R_{a}$ & $0.0064 \Omega$ \\
\hline$k_{t}$ & $8.79 \mathrm{Nm} / \mathrm{Amp}$ \\
\hline$k_{e}$ & $8.79 \mathrm{~V} / \mathrm{rad} / \mathrm{sec}$ \\
\hline \multicolumn{2}{|c}{ TABLE I }
\end{tabular}

PARAMETERS FOR EACH OF THE DC MOTORS IN THE TEST FACILITY USED IN THE PYTHON SIMULATION.

used in the test facility shown in Fig. 4. These parameters appear in Table I. Both the motors are separately excited fully compensated DC machines.

\section{A. Case $1-J_{\mathrm{em}}=1.3 J_{d}, B_{\mathrm{em}}=B_{d}$}

In this case the inertia is a bit higher that the actual inertia of the drive machine, and the friction is the same as the drive machine $\left(B_{d}=B_{l}=2.5 \mathrm{Nm} / \mathrm{r} / \mathrm{s}\right)$. The simulation has been set-up with a repeating speed profile, and similarly the disturbance torques follow a repeating profile. The disturbance torques are to simulate torque pulsations that are experienced during an electric rope shovel dig cycle as the dig bucket hits rocks and other obstructions. The control period for the outer emulation controller is $T_{s}=100 \mathrm{msec}$, this being a constraint

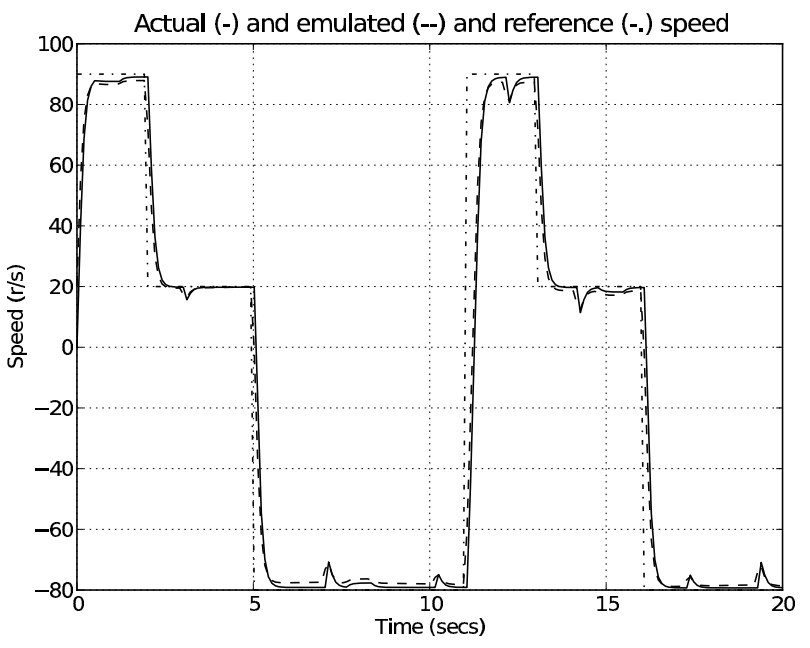

Fig. 9. Case 1: Actual (-), emulated (-) and reference (-.) speeds with $J_{\mathrm{em}}=1.3 J_{D}$ and $T_{s}=100 \mathrm{msec}$.

of the real hardware due to the way that signals can be sent to the drives. The current control is implemented with a period of $3.33 \mathrm{msec}$ (set by the rate at which the thyristor drive can change the applied voltage).

Fig. 9 shows the speed response of the system. The solid line is the actual speed of the dynamometer, and the dashed line is the emulation model output (i.e. what the real response should be), and the dash-dot line is the speed reference into the system. As can be seen, the true speed closely follows the 


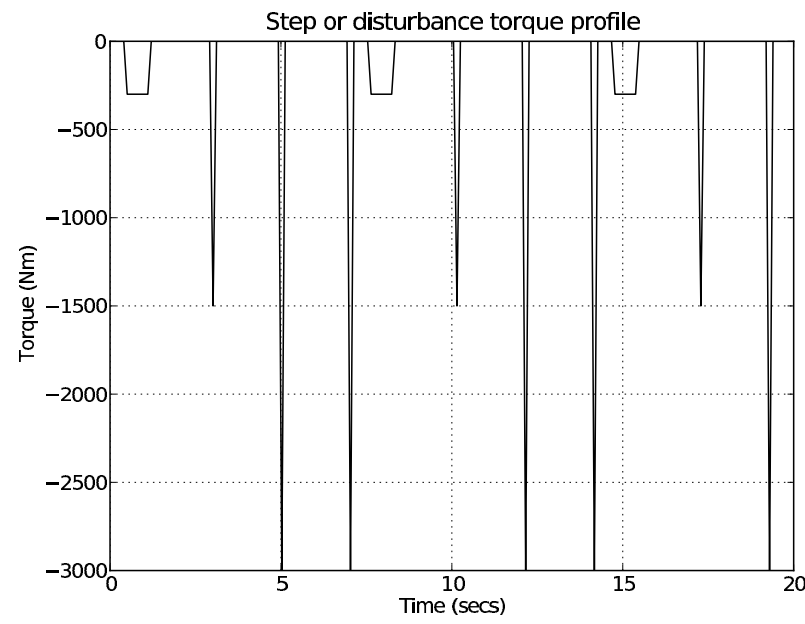

Fig. 10. Case 1: Torque disturbance profile.

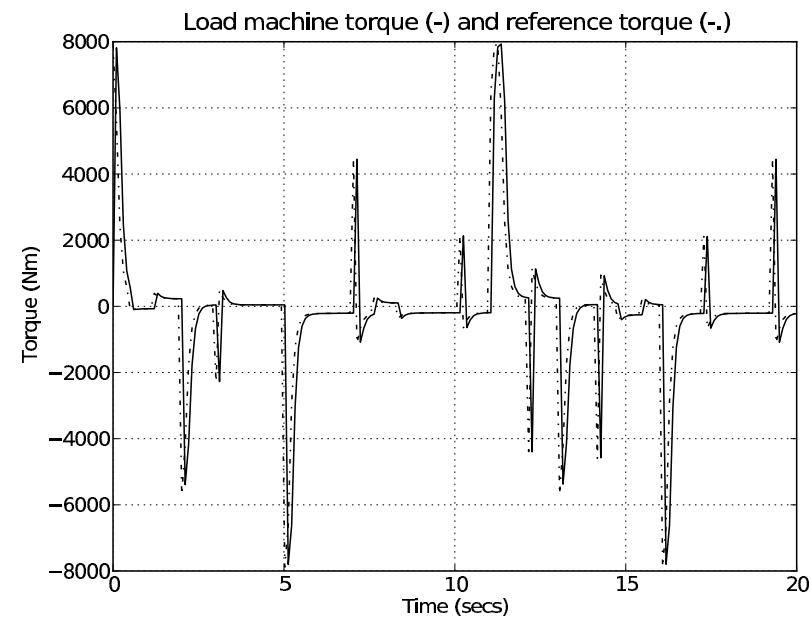

Fig. 11. Case 1: Load torque, actual (-) and reference (-.) with $J_{\mathrm{em}}=1.3 J_{D}$ and $T_{s}=100 \mathrm{msec}$.

desired emulated response. If the control period is shortened the plots actually sit on top of each other. The blips on the speed are due to the torque pulsations. Some of these are quite large, as can be seen from the disturbance torque profile in Fig. 10.

The final plot shown in Fig. 11 is the load machine torque. Again one can see that the profile of the desired torque is accurately followed, albeit with some digital delay. In this case the delay does not matter, only the accuracy of the trajectory tracking is important.

\section{CONClusions AND CONTRIBUtions}

The main contribution of this paper is the development and simulation of a control system for a large scale dynamic dynamometer that allows the emulation of the digging conditions experienced in mining applications, and in particular, electric rope shovel operations. The simulation studies have demonstrated that the control approach does an excellent job of the emulation. In addition it is very tolerant of the low control rates that will be used in the experimental system.
This system will allow, in a controlled environment, thorough testing of various wear mechanisms in DC motors used in electric rope shovels and draglines. A future paper will present experimental results of the dynamic dynamometer implemented on the $2.2 \mathrm{MW}$ dynamometer shown in the paper.

\section{REFERENCES}

[1] Z.H. Akpolat, G.M. Asher, and J.C. Clare. Experimental dynamometer emulation of nonlinear mechanical loads. Industry Applications, IEEE Transactions on, 35(6):1367-1373, Nov/Dec 1999.

[2] J. Arellano-Padilla, G.M. Asher, and M. Sumner. Control of an ac dynamometer for dynamic emulation of mechanical loads with stiff and flexible shafts. Industrial Electronics, IEEE Transactions on, 53(4):12501260, June 2006.

[3] R.E. Betz, H.B. Penfold, and R.W. Newton. Local vector control of an ac drive system load simulator. In Control Applications, 1994., Proceedings of the Third IEEE Conference on, pages 721-726 vol.1, Aug 1994.

[4] Z. Hakan Akpolat, G.M. Asher, and J.C. Clare. Dynamic emulation of mechanical loads using a vector-controlled induction motor-generator set. Industrial Electronics, IEEE Transactions on, 46(2):370-379, Apr 1999.

[5] G. Mirzaeva, R.E. Betz, and T.J. Summers. Evaluation of current density in dc motor brushes for mining machines based on air gap field measurement. In Industry Applications Conference, 2009. 44th IAS Annual Meeting. Conference Record of the, pages 1-8, 4-8 Oct 2009.

[6] G. Mirzaeva, R.E. Betz, T.J. Summers, and I. Marxsen. Development of a unique dc motor test facility in the hunter valley. In Proc. of the Australian Mining Technology Conference: Smart Technologies for Sustaining the Minerals Boom. 16-18 September 2008, Twin Waters, QLD, Australia, pages 95-106, 2008.

[7] P. Sandholdt, E. Ritchie, J.K. Pedersen, and R.E. Betz. A dynamometer performing dynamical emulation of loads with nonlinear friction. In Industrial Electronics, 1996. ISIE '96., Proceedings of the IEEE International Symposium on, volume 2, pages 873-878 vol.2, Jun 1996.

[8] Karl J. Åström and Bjön Wittenmark. Computer Controlled Systems - Theory and Design. Prentice-Hall Information and Systems Science Series. Prentice-Hall, 1984. ISBN 0-13-164319-3.

[9] R. Wendel Newton, R.E. Betz, and H.B. Penfold. A dynamic dynamometer for testing variable speed drives. In Industry Applications Society Annual Meeting, 1994., Conference Record of the 1994 IEEE, pages 538544 vol.1, Oct 1994. 